\title{
A REVIEW PAPER ON INDOOR NAVIGATION SYSTEM
}

\author{
Ankita Sandip Chipade, Rutik Hanish Mehta, Anish Charles Roham, Aarya Ramchandra Naik \\ Information Technology, MSBTE, Pune, Maharashtra, India \\ Mrs.R.B. Gurav \\ Information Technology, MSBTE, Pune, Maharashtra, India
}

\begin{abstract}
With the rapid development of smartphone industry, various positioning-enabled sensors such as GPS receivers, accelerometers, gyroscopes, digital compasses, cameras, $\mathrm{Wi}-\mathrm{Fi}$ and Bluetooth have been built in smartphones for communication, entertainment and location-based services. Smartphone users can get their locations fixed according to the function of GPS receiver. This is the primary reason why the huge demand for realtime location information of mobile users has been unprecedented in recent years. However, the GPS receiver is often not effective in indoor environments due to the signal attenuation and multipath effects, although it as the major positioning devices have a powerful accuracy for outdoor positioning. This research investigates other built-in GPS and develops methods for improving the accuracy of indoor positioning. Combination with the wireless network, it can be a viable alternative solution for the indoor positioning purposes of the smartphone users. The main advantage of this solution is that it can be deployed with a minimal cost, as no specialized hardware is necessary for setting up the system. However, challenges remain for this solution due to complex indoor environment involved and extensive calibration data overhead
\end{abstract}

Keywords- Wi-Fi Router, Detecting location.

\section{INTRODUCTION}

The software packages used for implementing the system use Android SD and languages used in the proposed system are php in server side, java in client side. Android is an operating system based on Linux with a Java programming interface. The Android Software Development Kit (Android SDK) provides all necessary tools to develop Android applications. This includes a compiler, debugger and a device emulator, as well as its own virtual machine to run Android programs. Android Software Development Kit (Android SDK) with Eclipse ADT is used for client side or for main application implementation. SDK 19 is used to develop the application. Initially, home page will be displayed in the application. When the user inside the campus, can view their current location. The user request the desired location in the map application. It shows the current and desired location with floor details. Using this application, user can know staff and event details. The user can request the staff or event details to the server. There is no effective features for finding the location inside the buildings. In the application, using the indoor location based services is used to find the current location of the mobile clients. Indoor Location Based Services is the extension of location based services. It is used for tracking the location inside the buildings or campus. Indoor Atlas android SDK is used for indoor navigation. The SDK offers the features like the indoor positioning with higher accuracy and obtaining floor level. In Indoor Atlas to track the desired location then update the floor details for desired location and after fixing the route inside the buildings.

\section{PROPOSED METHODOLOGY}

We propose an enhanced particle filter to fuse range information estimation as well as floor plan information for indoor tracking. We incorporate a double re-sampling method in the particle filter. This double re-sampling is a continuous and asynchronous process, which is able to mitigate the tracking errors caused by unstable location readings and low sampling frequency experimented in Wi-Fi sensors of commercial smart phones. We implement and evaluate a real-time terminal-based positioning system, which runs our proposed tracking algorithms on commercial smart phones. Our solution does not require any interaction with an additional external server. We conduct a set of extensive experiments to evaluate the system in complex indoor environments with long tracking paths.

\section{Algorithm}

For the proposed work and user location finding approach we solve using Triangulation problem. 
Basically triangulation is a term originally used in the navigation circles to obtain multiple reference point and locate an unknown position.

The triangulation is based on the following phases:

- With the powers of three access points that receive the user, is created an equations system, representing three circles.

- The system is solved to obtain a set of points, called triangulation points.

- Each triangulation point is considered a vertex of a triangle.

- All possible triangles are formed and the areas are calculated to compare them.

- The center of the triangle with the smallest area is taken as the estimate location of the user.

The calculation of the distance between the fingerprinting data base points and the user instant point is done as follows:

Current_signal_power $=$ power vector obtained by scanning the Access points signal power on a certain position.

FP_data $=$ database obtained during the fingerprinting process.

FP_points = power vector with the obtained results in each point.

$\mathrm{K}$-minimums $=$ vector with the three nearest points.

$$
\mathrm{d}(\mathrm{p}, \mathrm{pi})=\frac{1}{\mathrm{M}} * \sqrt{\sum_{\mathrm{J}=1}^{\mathrm{M}}\left(\mathrm{RSSI}_{\mathrm{j}} \mathrm{j}(\mathrm{X}, \mathrm{Y})-\mathrm{RSSI}_{\mathrm{j}}(\mathrm{Xj}, \mathrm{Yj})\right)^{2}}
$$

Where:

- $\mathrm{d}(\mathrm{p}, \mathrm{pi})=$ distance(Current_signal_power, FP_points)

- $\quad M=$ number of points in the fingerprinting database.

- RSSI_1 would be the power received by the AP1 in a certain point $(\mathrm{X}, \mathrm{Y})$.

- User_location = barycentre ( $\mathrm{k}$-minimums)

*RSSI $=$ received signal strength indication.

Where barycentre of the k-minimums is:

$$
\mathrm{P}=\frac{\sum_{\mathrm{J}=1}^{\mathrm{K}} \frac{1}{\operatorname{dist}(\mathrm{Z}, \mathrm{Zj})} * \mathrm{Xj}}{\sum_{\mathrm{j}=1}^{\mathrm{k}} \frac{1}{\operatorname{dist}(\mathrm{Z}, \mathrm{Zj})}}
$$

Being $\mathrm{P}=$ user location.

\section{SYSTEM DESIGN}

Block Diagram-

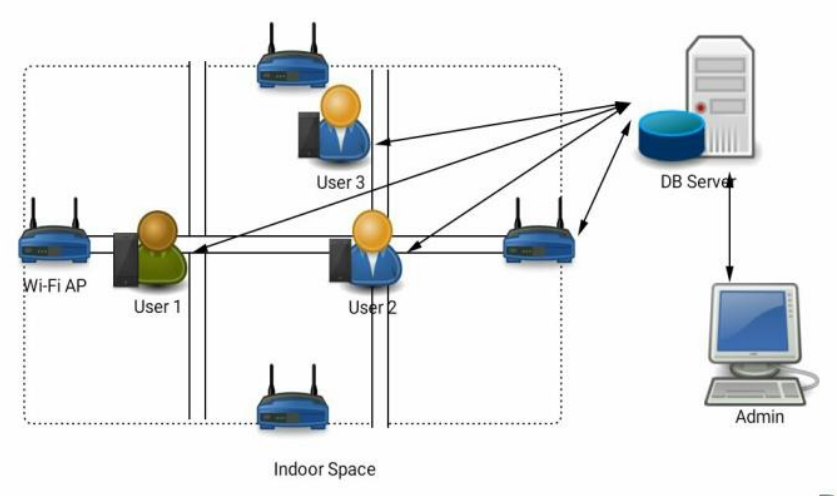

IV. SYSTEM REQUIREMENTS

Software Requirements:

1. Operating system: Windows XP/7 Higher

2. Programming Language: Android, Java

3. Tools: Android Studio, Heidi SQL, JDK 1.7 or higher.

Hardware Requirements:

1. System: Intel i5-9400f

2. Hard Disk: 1 TB

3. Ram: 8 GB

4. Monitor: 1920x1080p 60hz

\section{PROBLEMS AND CHALENGES}

In this part, we discuss the previous problems and challenges that occurred during the implementation of indoor navigation system.

A. Accuracy

Accuracy roughly refers to the difference between the estimated position and the actual one; as this difference could change depending on the conditions, it is rather a statistical distribution, 
which should be expressed in terms of parameters like a distance and a percentage, such as "less than one-meter error in 95 percent of cases," though authors rarely express accuracy in these terms.

B. Coverage

Coverage is the territorial extension in which the system can locate a user or object. Although some technologies may offer extensive coverage in an ideal environment, when these are used indoors, their coverage may be limited by environmental factors. An IPS may locate a person or object in a range of meters or even locate them at different levels inside a building.

C. Cost

In this survey, cost is determined based on two parameters. The first is installation and maintenance cost. The second is cost for each end user. We identify that IPS that make use of existing infrastructures, as well as systems that reuse technology that exists in the indoor environments (e.g., Wi-Fi access points) or those that are carried by the user (e.g., mobile devices), require low (L) investments for installation and maintenance and low cost of the service for the user.

\section{COMPARATIVE STUDY}

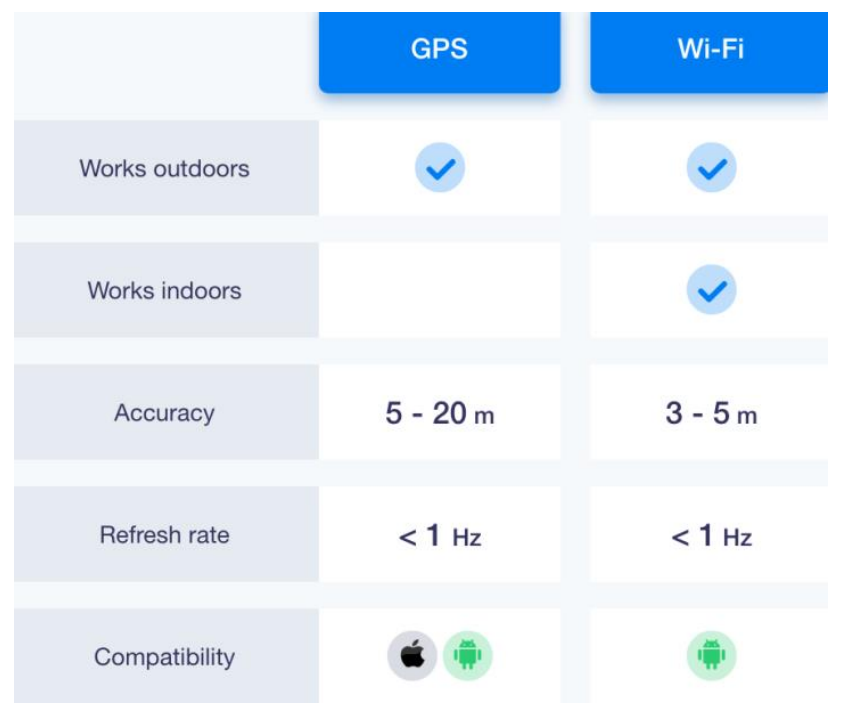

\section{CONCLUSION}

We proposed an evaluation framework which combines the key aspects of indoor navigation for investigating mobile indoor navigation systems. Based on this evaluation framework, we gave a comparison and analysis of the current mobile indoor navigation systems, and then identified some challenges which require further research and development.

We can draw the conclusion that indoor navigation systems are still on an early development stage.

\section{REFERENCES}

[1] Dominik Aufderheide and Werner Krybus. Towards Real-Time Camera Egomotion Estimation and Three-Dimensional Scene Acquisition from Monocular Image Streams. In International Conference on Indoor Positioning and Indoor Navigation (IPIN), pages 1-10. IEEE, 2010.

[2] Luiz FF Belussi and Nina ST Hirata. Fast QR Code Detection in Arbitrarily Acquired Images. In 24th SIBGRAPI Conference on Graphics, Patterns and Images, pages 281-288. IEEE, 2011.

[3] Martin A Fischler and Robert C Bolles. Random Sample Consensus: A Paradigm for Model Fitting With Applications to Image Analysis and Automated Cartography. Communications of the ACM, 24(6):381-395, 1981.

[4] Richard I Hartley. Euclidean Reconstruction From Uncalibrated Views. In Applications of invariance in computer vision, pages 235-256. Springer, 1993.

[5] Jinwook Huh, Woong Sik Chung, Sang Yep Nam, and Wan Kyun Chung. Mobile Robot Exploration In Indoor Environment Using Topological Structure with Invisible Barcodes. ETRI, 29(2):189-200, 2007.

[6] Nathan Koenig and Andrew Howard. Design and Use Paradigms for Gazebo, an Open-Source Multi-Robot Simulator. In 2004 IEEE/RSJ International Conference on Intelligent Robots and Systems (IROS 2004), volume 3, pages 2149-2154. IEEE, 2004. 